\title{
IL NUOVO SISTEMA ELETTORALE ITALIANO: QUALI OPPORTUNITÀ?
}

\section{di Roberto D'Alimonte e Alessandro Chiaramonte}

Il sistema elettorale è un complesso di regole che governano la traduzione delle preferenze in voti e dei voti in seggi (Rae $1971,14) \mathrm{e}$, in quanto tale, costituisce al contempo un vincolo ed una risorsa per tutti coloro - elettori, partiti, candidati - i quali, a vario titolo, concorrono a determinare la rappresentanza politica. In altre parole, esso contribuisce a modellare la struttura delle opportunità di quegli attori, il tipo di competizione che prevale a livello del sistema partitico e, in certi casi, l'espressione stessa delle preferenze elettorali.

La riforma del sistema elettorale italiano, con il passaggio dal principio della rappresentanza proporzionale (pura) ad un sistema prevalentemente maggioritario ${ }^{1}$, pone ai partiti delicate questioni di scelta di strategia elettorale, di alleanze politiche, di gestione delle candidature, e, agli elettori, di utilizzazione del voto. $\grave{E}$ da queste scelte, indubbiamente condizionate dalle caratteristiche tecniche del sistema elettorale, che dipenderanno gli effetti complessivi della riforma. Ciò non significa che le

Desideriamo ringraziare Antonio Agosta, Augusto Barbera, Stefano Bartolini, Mario Caciagli e Leonardo Morlino per i loro commenti ad una prima versione di questo lavoro.

\footnotetext{
1 Si è sostenuto che, a partire dagli anni venti-trenta, le riforme elettorali che hanno sancito l'abbandono del principio proporzionale in favore del principio maggioritario (o viceversa), per quanto infrequenti, sono state realizzate solo in coincidenza con le fasi di instaurazione di un regime - si veda in tal senso Nohlen $(1989,217)$. Non è questo il caso, così come non lo sono stati quelli relativi al passaggio da un sistema maggioritario puro ad un sistema misto (ma comunque fondato sulla logica propria alla proporzionale) verificatesi in Francia nel 1986 e di recente in Nuova Zelanda. La riforma in questi due paesi si spiega per il primo con la sopravvenuta convenienza al cambiamento da parte del principale partito di governo e per il secondo con l'esito di un voto referendario espresso in opposizione ai maggiori partiti. In tal senso, pur partendo da sistemi diversi, quanto accaduto in Italia è assimilabile all'esperienza neozelandese. Data comunque la resistenza offerta in tutte queste esperienze al mutamento delle regole, non pare venir meno la constatazione di una sostanziale stabilità dei sistemi elettorali, per $i$ motivi della quale si veda Reeve e Ware (1992, 10-14).
} 
nuove regole determineranno il nuovo sistema partitico. Più semplicemente, ne influenzeranno la formazione attraverso gli incentivi offerti a determinati comportamenti. L'obbiettivo di questo lavoro è, dunque, quello di mettere in evidenza questa struttura di incentivi mediante un'analisi puntuale delle proprietà dei meccanismi del sistema elettorale e di alcuni dei loro possibili effetti sulla struttura della competizione politica.

\section{La nuova legge elettorale per la Camera dei deputati}

Il Parlamento ha approvato il 4 agosto scorso la nuova disciplina per l'elezione della Camera e del Senato², in conseguenza del risultato referendario del 18 aprile ${ }^{3}$. Essa è il frutto di un complesso gioco di accordi e di veti incrociati tra gli schieramenti presenti nell'arena parlamentare e costituisce, pertanto, una soluzione di compromesso. L'esito di tale compromesso è un sistema elettorale misto maggioritario-proporzionale che si configura come un unicum nel panorama dei regimi politici democratici.

Ai fini della maggiore chiarezza espositiva, è utile illustrare gli aspetti fondamentali della nuova normativa in riferimento alle singole variabili che definiscono il concetto di sistema elettorale ${ }^{4}-\mathrm{la}$ struttura del voto, la dimensione dei collegi e la formula elettorale - e alle disposizioni legislative di contorno 5 che ad esse direttamente ineriscono. Cominceremo dal sistema di elezione della $\mathrm{Ca}$ mera dei deputati che, come vedremo, offre risvolti più problematici; la nuova legislazione elettorale del Senato e le differenze con quella della Camera saranno trattate in una sezione successiva.

2 La L. 4 agosto 1993, n. 277, recante «Norme per l'elezione della Camera dei deputati» (pubblicata nella Gazzetta Ufficiale - serie generale - n. 183 del 6 agosto 1993), e la L. 4 agosto 1993, n. 276, recante «Norme per l'elezione del Senato della Repubblica» (pubblicata in id.), formalmente apportano modificazioni - rispettivamente - al D.P.R. 30 marzo 1957 (e successive modificazioni) e alla L. 6 febbraio 1948, n. 29 (e successive modificazioni). Al momento della stesura di questo articolo non erano ancora stati pubblicati i regolamenti di attuazione delle due leggi.

3 Il 18 aprile 1993 gli elettori italiani si sono pronunciati a larghissima maggioranza a favore dell'abrogazione di quelle norme della legge elettorale per il Senato che impedivano al sistema elettorale di funzionare secondo il principio maggioritario-uninominale. Se la nuova legislazione elettorale del Senato, ed anche quella della Camera, accolgano in pieno lo spirito di quel voto non è facile a dirsi.

4 Si fa qui riferimento alla definizione operazionale di Rae $(1971,16)$ e di Taagepera e Shugart $(1989,19)$. Una puntualizzazione sulle variabili sottese al concetto di sistema elettorale è presente in Blais (1988).

s Sono disposizioni legislative di contorno le norme sull'elettorato attivo e passivo, le modalità di presentazione delle liste e delle candidature, la costituzione delle sezioni elettorali, le disposizioni penali. 
Collegi e circoscrizioni. Il territorio nazionale è suddiviso in 26 circoscrizioni elettorali, ognuna delle quali dispone di un numero di seggi da ripartire in base agli abitanti'. All'interno di ciascuna circoscrizione il $75 \%$ dei seggi complessivi viene assegnato a singoli candidati in altrettanti collegi uninominali ${ }^{7}$, mentre il restante $25 \%$ è attribuito a liste concorrenti nella stessa circoscrizione (Tab. 1).

TAB. 1 Circoscrizioni elettorali

\begin{tabular}{|c|c|c|c|c|c|}
\hline$n$. & Circoscrizioni & Province & $\begin{array}{l}\text { Seggi } \\
\text { tot. }\end{array}$ & $\begin{array}{l}\text { Seggi } \\
\text { unin. }\end{array}$ & $\begin{array}{l}\text { Seggi } \\
\text { prop. }\end{array}$ \\
\hline 1 & Piemonte 1 & Torino & 25 & 19 & 6 \\
\hline 2 & Piemonte 2 & $\begin{array}{l}\text { Vercelli, Novara, Alessandria, Asti, } \\
\text { Cuneo, Biella, Verbano-Cusio- } \\
\text { Ossola }\end{array}$ & 23 & 17 & 6 \\
\hline 3 & Lombardia 1 & Milano & 41 & 31 & 10 \\
\hline 4 & Lombardia 2 & $\begin{array}{l}\text { Varese, Como, Sondrio, Lecco, } \\
\text { Bergamo, Brescia }\end{array}$ & 42 & 32 & 10 \\
\hline 5 & Lombardia 3 & Pavia, Cremona, Mantova, Lodi & 15 & 11 & 4 \\
\hline 6 & Trentino-Alto Adige & & 10 & 8 & 2 \\
\hline 7 & Veneto 1 & Verona, Vicenza, Padova, Rovigo & 29 & 22 & 7 \\
\hline 8 & Veneto 2 & Venezia, Treviso, Belluno & 20 & 15 & 5 \\
\hline 9 & Friuli-Venezia Giulia & & 13 & 10 & 3 \\
\hline 10 & Liguria & & 19 & 14 & 5 \\
\hline 11 & Emilia-Romagna & & 43 & 32 & 11 \\
\hline 12 & Toscana & & 39 & 29 & 10 \\
\hline 13 & Umbria & & 9 & 7 & 2 \\
\hline 14 & Marche & & 16 & 12 & 4 \\
\hline 15 & Lazio 1 & Roma & 42 & 32 & 10 \\
\hline 16 & Lazio 2 & Viterbo, Rieti, Latina, Frosinone & 15 & 11 & 4 \\
\hline 17 & Abruzzi & & 14 & 11 & 3 \\
\hline 18 & Molise & & 4 & 3 & 1 \\
\hline 19 & Campania 1 & Napoli & 33 & 25 & 8 \\
\hline 20 & Campania 2 & $\begin{array}{l}\text { Caserta, Benevento, Avellino, } \\
\text { Salerno }\end{array}$ & 29 & 22 & 7 \\
\hline 21 & Puglia & & 45 & 34 & 11 \\
\hline 22 & Basilicata & & 7 & 5 & 2 \\
\hline 23 & Calabria & & 23 & 17 & 6 \\
\hline 24 & Sicilia 1 & $\begin{array}{l}\text { Palermo, Trapani, Agrigento, } \\
\text { Caltanisetta }\end{array}$ & 27 & 20 & 7 \\
\hline 25 & Sicilia 2 & $\begin{array}{l}\text { Messina, Catania, Ragusa, Siracusa } \\
\text { Enna }\end{array}$ & 28 & 21 & 7 \\
\hline 26 & Sardegna & & 18 & 14 & 4 \\
\hline & (Valle d'Aosta) & & 1 & 1 & 0 \\
\hline Tot & Italia & & 630 & 475 & 155 \\
\hline
\end{tabular}

6 La Valle d'Aosta è un'ulteriore circoscrizione elettorale costituita comunque in collegio uninominale e per la quale valgono le norme precedenti.

$7 \mathrm{Se}$ il calcolo del $75 \%$ non dà un numero intero, l'arrotondamento è effettuato per eccesso qualora la cifra decimale sia uguale o superiore a 50 . Ciascun collegio ha in media 120.000 abitanti. 
Una apposita commissione è stata istituita per la determinazione dei collegi uninominali in base ai principi direttivi della coerenza territoriale, dell'omogeneità economico-sociale e storico-culturale, e della popolazione residente. A quest'ultimo riguardo la legge prevede che la popolazione di ciascun collegio possa scostarsi dalla media della popolazione dei collegi della circoscrizione non oltre il dieci per cento in eccesso o in difetto, salvo nelle zone in cui siano presenti minoranze linguistiche, nel qual caso la tolleranza è del quindici per cento. La nomina di una commissione per la revisione dei collegi elettorali è prevista ad ogni inizio di legislatura.

Candidature e contrassegni. Nel collegio uninominale ogni candidato (candidati di collegio) deve obbligatoriamente collegarsi ad almeno una lista presente a livello circoscrizionale; il collegamento risulta da una dichiarazione rilasciata dal candidato stesso e sottoscritta da un rappresentante della lista. In caso di collegamenti con più liste, questi devono essere i medesimi in tutti $\mathrm{i}$ collegi della circoscrizione. Ciascun candidato può presentarsi in un solo collegio uninominale e, ai fini della votazione in quella sede, è accompagnato da un minimo di uno ad un massimo di cinque contrassegni. Le liste concorrenti nella circoscrizione sono rappresentate da un contrassegno e da un elenco di candidati (candidati di lista); il numero di detti candidati non può essere superiore ad un terzo ${ }^{8}$ dei seggi spettanti alla circoscrizione ai fini del riparto proporzionale. Ogni candidato può essere incluso in liste aventi il medesimo contrassegno in non più di tre circoscrizioni. Anche i candidati di collegio possono far parte delle candidature di lista, ma solo di liste collegate. Le liste recanti più di un nome sono formate da candidati e candidate in ordine alternato?.

Struttura del voto. Le consultazioni si svolgono in un turno unico. Ogni elettore dispone di due voti da esprimere in altrettante schede, che, pertanto, possono essere disgiunti. Un primo voto è finalizzato all'elezione di un candidato nel collegio uninominale. Il secondo voto viene attribuito ad una lista nella cir-

8 L'eventuale arrotondamento è effettuato per eccesso. In base alla ripartizione dei seggi alle circoscrizioni conseguente ai dati del censimento del 1991, il numero massimo dei candidati di lista da poter presentare varierà da 1 a 4 .

- Non è ancora chiaro se l'ordine alternato uomo-donna nell'elenco dei candidati di lista debba essere considerato vincolante. Dalla lettera della legge sembrerebbe comunque di sì. 
coscrizione; a tal riguardo l'elettore non può esprimere oltre al voto per la lista anche un voto di preferenza, trattandosi di liste bloccate.

Formula elettorale e collegi uninominali. In ciascun collegio uninominale viene eletto il candidato che ottiene il più alto numero di suffragi espressi. La conquista del seggio è pertanto determinata secondo la regola della maggioranza relativa (plurality).

Ripartizione proporzionale dei seggi tra le liste. Il computo dei seggi spettanti alle liste avviene a livello nazionale (Tab. 2). A tal fine è presente una clausola di sbarramento: sono esclusi i partiti che non abbiano ottenuto almeno il $4 \%$ dei voti validamente espressi sul totale delle circoscrizioni. Per i restanti si applica il metodo del quoziente naturale e dei più alti resti.

TAB. 2 Formule per la ripartizione proporzionale dei seggi

\begin{tabular}{|c|c|c|}
\hline \multicolumn{2}{|c|}{ - Soglia di sbarramento: } & $S K=\left(\Sigma_{i j} V_{i j}\right) 4 / 100$ \\
\hline \multicolumn{2}{|c|}{ - Cifra elettorale circoscrizionale: } & $\mathrm{CEC}_{\mathrm{kj}}=\mathrm{V}_{\mathrm{kj}}-\mathrm{V}(\mathrm{sc})_{\mathrm{kj}}$ \\
\hline \multicolumn{2}{|c|}{ - Quoziente elettorale circoscrizionale: $\mathrm{QEC}_{\mathrm{i}}=\left(\Sigma_{\mathrm{k}} \mathrm{CEC}_{\mathrm{kj}}\right) / \mathrm{S}_{\mathrm{i}}$} & $\mathrm{QEC}_{\mathrm{i}}=\left(\Sigma_{\mathrm{k}} \mathrm{CEC}_{\mathrm{kj}}\right) / \mathrm{S}_{\mathrm{i}}$ \\
\hline \multicolumn{2}{|c|}{ - Cifra elettorale nazionale: } & $\mathrm{CEN}_{k}=\Sigma_{\mathrm{i}} \mathrm{CEC}_{\mathrm{kj}}$ \\
\hline \multicolumn{2}{|c|}{ - Quoziente elettorale nazionale: } & $\mathrm{QEN}=\left(\Sigma_{k} \mathrm{CEN}_{k}\right) / \mathrm{S}_{n}$ \\
\hline \multicolumn{3}{|c|}{ Legenda: } \\
\hline $\mathrm{V}_{\mathrm{ij}}$ & \multicolumn{2}{|c|}{ voti della lista i-esima nella circoscrizione j-esima* } \\
\hline $\mathrm{V}_{\mathrm{kj}}^{\prime}$ & \multicolumn{2}{|c|}{ voti della lista $k$-esima nella circoscrizione j-esima* } \\
\hline$V(s c)_{k j}$ & \multicolumn{2}{|c|}{$\begin{array}{l}\text { voti da detrarre in virtù dello scorporo alla lista } k \text {-esima nella circoscrizione } \\
\text { j-esima }\end{array}$} \\
\hline$S_{i}$ & \multicolumn{2}{|c|}{ seggi della circoscrizione j-esima da assegnarsi proporzionalmente } \\
\hline$S_{n}$ & \multicolumn{2}{|c|}{ seggi nazionali da assegnarsi proporzionalmente $\left(S_{n}=\Sigma_{i} S_{i}\right)$} \\
\hline SK & \multirow{2}{*}{\multicolumn{2}{|c|}{$\begin{array}{l}\text { soglia di sbarramento } \\
\text { cifra elettorale circoscrizionale della lista } k \text {-esima nella circoscrizione } j \text { - } \\
\text { esima }\end{array}$}} \\
\hline $\mathrm{CEC}_{\mathrm{kj}}$ & & \\
\hline $\mathrm{CEN}_{\mathrm{k}}$ & \multicolumn{2}{|c|}{ cifra elettorale nazionale della lista k-esima } \\
\hline $\mathrm{QEC}_{\mathrm{i}}$ & \multicolumn{2}{|c|}{ quoziente elettorale circoscrizionale della circoscrizione j-esima } \\
\hline QEN & \multicolumn{2}{|l|}{ quoziente elettorale nazionale } \\
\hline
\end{tabular}

* La lista i-esima è ogni lista che ha conseguito voti; la lista k-esima è ogni lista (sottoinsieme delle liste $\mathrm{i}$-esime) che ha conseguito, in ambito nazionale, almeno il $4 \%$ dei voti.

Per la determinazione del quoziente elettorale nazionale si fa riferimento, in primo luogo, alle cifre elettorali circoscrizionali di ciascuna lista. Queste ultime corrispondono al totale dei voti validi attribuiti alla lista detratto, per ciascun collegio uni- 
nominale in cui è stato eletto un candidato collegato alla lista stessa, un numero di voti (aumentato di una unità) pari a quello conseguito dal candidato primo dei non eletti in quello stesso collegio; tale numero di voti non può comunque essere inferiore al $25 \%$ dei voti validii ${ }^{10}$. Qualora il candidato eletto sia collegato a più liste, la detrazione avviene pro quota in misura proporzionale alla somma dei voti proporzionali conseguiti da ciascuna di esse nell'ambito territoriale del collegio. E questo il meccanismo cosiddetto dello scorporo.

La somma delle cifre elettorali circoscrizionali delle liste aventi lo stesso contrassegno (ossia del singolo partito) ne costituisce la cifra elettorale nazionale. Il quoziente elettorale nazionale è il rapporto tra le cifre elettorali nazionali di tutte le liste che hanno superato la soglia di sbarramento e il totale dei seggi $\mathrm{da}$ ripartire in ragione proporzionale.

I seggi ripartiti a livello nazionale, poi, sono distribuiti alle liste nelle singole circoscrizioni in base ad una procedura piuttosto complessa. Immediatamente vengono assegnati $i$ seggi corrispondenti ai quozienti circoscrizionali ${ }^{11}$ interi conseguiti dalle liste. I seggi residui sono attribuiti seguendo la graduatoria decrescente delle parti decimali del quoziente di ciascuna lista, ma in modo tale da rispettare sia il numero di seggi spettanti ad ogni circoscrizione, che la distribuzione effettuata a livello nazionale. A tal fine le operazioni procedono dalla circoscrizione di minore ampiezza ed escludono di volta in volta le liste che abbiano esaurito la quota di seggi stabilita appunto in sede nazionale; se necessario, gli ulteriori eventuali seggi residui sono assegnati alle liste che ne hanno diritto nelle circoscrizioni in cui esse hanno i maggiori resti, utilizzando per primi i resti che non abbiano già dato luogo all'attribuzione di seggi.

Assegnazione ai candidati dei seggi proporzionalmente distribuiti. In ogni circoscrizione, la proclamazione degli eletti avviene, in ragione dei seggi spettanti a ciascun partito, a cominciare dai candidati di lista secondo l'ordine progressivo di presentazione. Se ciò non fosse sufficiente a coprire tutti i mandati cui la lista ha diritto, si procede in base alla graduatoria delle cifre

${ }^{10} \mathrm{Se}$, però, il candidato vincente nel collegio uninominale non ha superato la soglia del $25 \%$ dei voti validi, il numero di voti detratto alla lista ad esso collegata è pari al numero di voti conseguito dal candidato stesso nel collegio.

11 Il quoziente elettorale circoscrizionale è pari al rapporto tra la somma delle cifre elettorali circoscrizionali delle liste che in sede nazionale hanno superato la clausola di sbarramento e il numero dei seggi che la circoscrizione deve assegnare. 
individuali dei candidati non eletti nei collegi uninominali e collegati alla lista medesima. Tali cifre individuali sono la percentuale dei suffragi da essi ottenuti calcolata sul numero dei votanti $^{12}$ nei rispeitivi collegi. Un candidato di collegio collegato a più liste entra nella graduatoria di ciascuna di esse; nel caso che la sua cifra individuale gli consentisse di essere eletto in più di una lista, gli sarà attribuito il seggio di quella con la cifra elettorale più elevata.

\section{L'interdipendenza tra maggioritario e proporzionale}

Un'osservazione superficiale delle nuove regole elettorali può condurre all'ingannevole conclusione che in esse convivano due livelli del tutto distinti di ripartizione dei seggi e - quindi di competizione. Ossia, che l'impatto del sistema elettorale sulla rappresentanza costituisca l'esito ponderato degli effetti di due formule applicate in altrettanti contesti: il plurality, per l'attribuzione di tre quarti dei seggi complessivi, e il metodo del quoziente naturale e dei più alti resti (con soglia di sbarramento), per il restante quarto.

In realtà, alla possibilità di espressione di due voti in schede diverse non corrisponde un'analoga differenziazione delle arene competitive. Al di là degli esiti che - come vedremo più avanti - è possibile aspettarsi dalla coesistenza di criteri non omogenei per la distribuzione dei seggi, infatti, la legge elettorale dispone di congegni che interconnettono la competizione dei candidati nei collegi uninominali e delle liste circoscrizionali (a dispetto del doppio voto). In particolare se ne possono individuare quattro:

1) il candidato di collegio deve essere collegato ad almeno una lista presente in sede circoscrizionale ${ }^{13}$;

2) il candidato di collegio può figurare anche tra i candidati di lista (della lista collegata);

3) l'elezione di un candidato di collegio, in virtù dello scorporo, determina per la lista o per le liste ad esso collegata/e una perdita di voti ai fini della attribuzione proporzionale dei seggi;

12 E non sul numero complessivo dei voti validamente espressi.

${ }_{13}$ Ciò significa l'impossibilità di candidature svincolate dal riferimento ad una lista; il che potrebbe comunque non impedire del tutto a candidati indipendenti di competere nei collegi. Questi ultimi, infatti, si collegherebbero a liste circoscrizionali da loro create, per la presentazione delle quali è necessaria la raccolta di un numero di sottoscrizioni che nella peggiore delle ipotesi è di 4.000 . 
4) i seggi acquisiti da una lista mediante il riparto proporzionale possono essere appannaggio di candidati di collegio perdenti collegati alla lista medesima.

In definitiva, ci troviamo di fronte ad un sistema elettorale ibrido, di difficile classificazione, che non può essere definito tout court per tre quarti maggioritario-uninominale e per un quarto proporzionale. Anche perché la componente maggioritaria non accoglie completamente la meccanica del plurality system, che invece parrebbe evocare. Vi è, infatti, una sostanziale diversità di principio riguardo ai collegi uninominali.

Collegi uninominali sono presenti in sistemi elettorali incardinati sia sulla logica proporzionalistica che su quella maggioritaria. Nei primi mascherano una dinamica per la quale l'esito del voto dipende da altri livelli territoriali di ripartizione dei seggi, favorendo comunque un più ravvicinato rapporto candidati-elettori ${ }^{14}$. Nel secondo caso sono funzionali alla regola del first past the post $\mathrm{o}$, altrimenti detta, del winner takes all, in base alla quale il vincitore conquista tutta la posta in palio (il seggio) e ai perdenti non sono concesse chances di rivincita (se non in altri collegi - nel caso in cui la legge lo consenta - ma alle stesse condizioni: sconfiggere tutti i concorrenti). Ebbene, le modalità di funzionamento dei collegi uninominali previste dall'attuale legislazione del nostro paese non sembrano conformarsi a nessuna delle due situazioni ora descritte: all'interno di detti collegi, né il voto si configura soltanto quale espressione di preferenza nei confronti di un candidato la cui elezione dipende dal consenso ricevuto dalla lista, né la competizione determina candidature definitivamente sconfitte. Da una parte il candidato che ottiene il maggior numero di suffragi conquista immediatamente il seggio; dall'altra i candidati perdenti conservano - sebbene limitatamente ad alcuni casi, in virtù del numero ridotto di seggi proporzionali disponibili in ciascuna circoscrizione - la possibilità di essere eletti. Nel caso in cui, infatti, alla/e lista/e loro collegate sia assegnato nella circoscrizione,

14 E' evidente il riferimento alla vecchia normativa italiana per l'elezione del Senato: il voto espresso ad un candidato era in realtà espresso alla lista ai fini della ripartizione proporzionale dei seggi spettanti a ciascuna circoscrizione regionale; tutt'al più esso poteva configurarsi (anche) come una sorta di alternativa al voto di preferenza. Anche il sistema di elezione del Bundestag tedesco prevede collegi uninominali che non interferiscono con la dinamica proporzionalistica della distribuzione complessiva dei seggi (ma che la configurano come un «sistema di rappresentanza proporzionale personalizzata»). 
grazie al riparto proporzionale, un numero di seggi superiore a quello delle candidature (di lista) - e nulla impedisce ai partiti di presentarne meno rispetto a quanto avrebbero diritto - sono gli stessi candidati sconfitti nel collegio uninominale, in base alle rispettive cifre individuali, a concorrere per il conseguimento di quei seggi residuali.

Di tutto questo è necessario tener conto allorché si affronta la questione delle possibili strategie di competizione adottate dagli attori politici.

\section{Meccanismi elettorali e strategie competitive dei partiti}

I sistemi elettorali che producono esiti notevolmente proporzionalistici - come quello in vigore in Italia sino a pochi mesi fa - hanno ridotte capacità manipolative ${ }^{15}$ nei confronti delle strategie elettorali dei partiti: il comportamento tipico di questi ultimi è di competere in proprio. Allearsi con altri partiti prima delle elezioni non solo non è necessario, ma può anche compromettere quella distinguibilità che costituisce una risorsa importante quando il numero dei partiti in competizione è molto elevato.

La modifica dei meccanismi elettorali, allorché si passa da un sistema proporzionale pressoché puro ad uno fondato sul principio maggioritario, impone, invece, una riconsiderazione delle strategie competitive ottimali. A tal fine il singolo partito, infatti, deve tener conto di due possibili obbiettivi: 1) la massimizzazione dei seggi per sé; 2) la massimizzazione dei seggi per quell'insieme (di cui è parte) di formazioni politiche tra cui è possibile prevedere futuri accordi in sede parlamentare. Tali obbiettivi non risultano più largamente coincidenti come accade in presenza di un sistema elettorale proporzionale, bensì potenzialmente in conflitto e, pertanto, implicano strategie alternative per il loro conseguimento ${ }^{16}$. In ogni caso le alleanze elet-

15 Il termine «capacità manipolative» è mutuato da Fisichella $(1970,121)$.

16 La distinzione tra questi obbiettivi si fonda sull'ipotesi che, soprattutto con sistemi elettorali maggioritari, il perseguimento dell'uno ovvero dell'altro produca esiti differenti. Chiariamo con un esempio. Poniamo che due partiti A e B decidano di gareggiare da soli e che la loro aspettativa in termini di seggi sia per il primo 100 e per il secondo 10. Se, dopo le elezioni, essi uniranno le loro forze avranno $110 \mathrm{seggi}$. Si ipotizzi, invece, che gli stessi due partiti decidano di allearsi in vista della consultazione elettorale e che il numero totale di seggi attesi sia di 120 , ma così suddivisi: 90 a candi- 
torali possono rivelarsi fondamentali, ma quale corso esse seguano dipende dalle scelte operate dai partiti di fronte alle mutate regole della competizione. Se il primo obbiettivo è prevalente sul secondo è probabile aspettarsi che le eventuali aggregazioni siano realizzate in specifiche circostanze e che siano mutevoli a seconda delle convenienze; in definitiva, che abbiano una natura sostanzialmente tattica. Se, al contrario, è il secondo obiettivo a prevalere sul primo sarebbe persino incentivante per le liste interessate adoperarsi ai fini di una vera e propria fusione, ossia della creazione di un nuovo soggetto politico; e, comunque, senza arrivare a tanto, i partiti si orienterebbero verso aggregazioni stabili a carattere nazionale. Ma obiettivi e strategie della competizione elettorale dipendono, per ciò che è da imputargli, dalla specifica configurazione del sistema elettorale - e dalla relativa struttura di incentivi - piuttosto che dalla circostanza che esso sia etichettato come maggioritario ${ }^{17}$.

Gli interrogativi ai quali intendiamo, dunque, dare una prima risposta sono i seguenti: il sistema elettorale italiano così come congegnato determina degli incentivi all'aggregazione tra liste a livello di collegi e/o in sede circoscrizionale? E, se così, quali tipi di aggregazione? In altre parole, in che modo il sistema elettorale contribuisce a determinare la struttura della competizione tra i partiti?

$\grave{E}$ ovvio che la possibilità di aggregazioni elettorali di varia natura - oltre che dagli obiettivi che si intendono perseguire dipende largamente da fattori quali la distanza ideologica tra le formazioni politiche, i rapporti di forza, il tipo di insediamento sul territorio (se concentrato o disperso), il potere negoziale, la previsione delle strategie dei partiti/candidati concorrenti, la fedeltà dei rispettivi elettorati, le contropartite, le finalità di lungo periodo, la qualità delle candidature, ed altri ancora; ma è innegabile che le regole elettorali abbiano capacità manipolative proprie e che la loro particolare connotazione determini di per sé un complesso di vincoli, entro i quali le opportunità d'azione dei partiti sono effettivamente influenzate dalle variabili ora menzionate. Appare dunque rilevante illustrare le possibilità

dati di A e 30 a quelli di B. Allora, mentre per B la strategia di aggregazione determina comunque un incremento dei propri seggi, per $A$ sarà necessario valutare quale dei due obiettivi sia prevalente, perché essi risultano alternativi.

17 Ammesso che il sistema elettorale della Camera dei deputati possa essere etichettato come tale. 
strategiche che la nuova legge elettorale offre ai partiti, a prescindere da alcuni o da gran parte dei fattori che entrano comunque in gioco nelle loro scelte effettive.

In prima approssimazione è plausibile affermare che in sede di collegio uninominale - ossia per il $75 \%$ circa dei seggi complessivi - sia fortemente disincentivata la proliferazione delle candidature, mentre a livello di ripartizione proporzionale, al di sopra della soglia del $4 \%$, tale vincolo, per le liste, non sussista più. $\mathrm{Da}$ ciò si desume che, ai fini della massimizzazione della probabilità di vittoria, $i$ partiti hanno interesse ad aggregarsi a sostegno di candidature comuni nei collegi uninominali, ed invece a «gareggiare» da soli per il voto di lista nelle circoscrizioni - a meno che non esista l'aspettativa del superamento della clausola di esclusione. Ma non è così semplice. Come già rilevato, tra le arene di competizione costituite dai collegi e dalle circoscrizioni esistono delle connessioni che rappresentano un impedimento all'eventualità di strategie partitiche completamente differenziate nei due livelli di voto.

Al livello dei seggi da assegnarsi proporzionalmente, la presenza della clausola di sbarramento del $4 \%$ di fatto costringe i partiti che non si aspettano di poterla superare ad un'unica scelta: per essi la via della sopravvivenza passa attraverso la incorporazione/fusione con altri partiti ${ }^{18}$. Non vi è invece alcun incentivo all'accorpamento in liste comuni per quelle compagini che prevedono per sé stesse un consenso tale da conseguire, con un certo margine di sicurezza, la rappresentanza. Nemmeno nel caso di quelle che abbiano realizzato alleanze elettorali in sede di collegi uninominali, dato che a livello del voto di lista possono mantenere la loro identità senza costi. A conferma dell'assenza di incentivi forti all'aggregazione tra liste, si consideri che i partiti che accedono alla rappresentanza ricevono un trattamento assolutamente parificato - ossia del tutto proporzionale ai reciproci rapporti di forza - quanto alla distribuzione dei seggi: l'adozione del metodo del quoziente naturale nell'ambito della circoscrizione unica coincidente con il territorio nazionale significa, infatti, l'annullamento di qualsiasi effetto distorsivo. Non è in questa sede, in definitiva, che possiamo aspettarci una

${ }^{18}$ L'entità della soglia non può essere valutata di per sè bassa, ma resta il fatto che molte delle attuali forze politiche medio-piccole - MSI, La Rete, Rifondazione Comunista, Alleanza Democratica, Verdi -, la potrebbero superare. 
significativa riduzione del numero di liste rappresentate e, quindi, una semplificazione del quadro politico.

Quanto alla competizione nei collegi uninominali, si è già sottolineata l'esistenza di meccanismi che la rendono almeno in parte dipendente dalla competizione nelle circoscrizioni. In base ad essi il voto di lista - ossia il voto attribuito più o meno a quegli stessi partiti presenti con il vecchio sistema elettorale ha di fatto un «peso specifico» superiore a quello del $25 \%$ dei seggi cui, secondo la legge, è confinato. In primo luogo perché i partiti presenti in sede circoscrizionale dispongono di strumenti idonei - tra cui il principale è la previsione del collegamento - a controllare ed orientare le strategie competitive dei candidati di collegio ${ }^{19}$. Inoltre perché l'espressione di preferenza nei confronti della lista, essendo scarsamente manipolata dal sistema, può conservare, al pari di quanto accaduto con la vecchia normativa, la funzione di termometro dei rapporti effettivi di forza in vista di possibili alleanze. Chiariamo meglio quest'ultimo punto.

I sistemi elettorali maggioritari forzano la confluenza dei piccoli partiti (con un elettorato disperso) in formazioni più grandi e competitive, pena la scomparsa ${ }^{20}$. Una scomparsa che sarebbe determinata, oltre che dall'incidenza sull'organizzazione di netti e ripetuti insuccessi, dallo spostamento del proprio elettorato verso l'espressione di un voto più influente. In presenza di un sistema elettorale come quello attuale per la Camera, invece, i partiti minori - oltre ad aver garantita la rappresentanza (comunque sopra il $4 \%$ dei suffragi) grazie alla ripartizione proporzionale di un quarto dei seggi complessivi - tenderanno a contrattare gli eventuali accordi elettorali non tanto in base alla propria quota attesa di seggi in sede uninominale, quanto a quella di consensi accordati mediante il voto di lista. Ciò è possibile proprio in virtù dell'opportunità concessa agli elettori di esprimere, con la seconda scheda, un voto al partito comunque più preferito. Dunque, i partiti minori hanno l'opportunità di richiedere compensazioni più consistenti di fronte

19 La competizione nei collegi, infatti, non è semplicemente una sfida tra singole personalità, bensì tra candidati comunque di partito; ossia tra candidati che sono stati selezionati e presentati nel collegio appunto in virtù di scelte operate da un partito o congiuntamente da più partiti alleati.

20 Il riferimento è evidentemente a piccoli partiti nazionali (con un elettorato non concentrato), non interessati al mantenimento di una propria identità attraverso la presentazione di candidati di bandiera. 
ad un'ipotesi di alleanza elettorale, ed hanno tutto sommato meno da perdere dalla sua mancata realizzazione, rispetto a quanto accadrebbe loro in presenza di un sistema maggioritario puro. L'esistenza stessa di un livello proporzionale di ripartizione dei seggi per il quale vige un voto separato, in sostanza, attenua gli effetti di un pur prevalente livello maggioritario offrendo un incentivo al mantenimento di forti identità di partito $^{21}$ e condizionando quindi le aggregazioni sia nella loro concreta fattibilità che nelle eventuali modalità di compimento. Il sistema elettorale allora non orienta i partiti alla formazione di compatti schieramenti alternativi, bensì verso alleanze locali ed eterogenee contrattate sulla base della rispettiva dotazione di voti proporzionali.

Ma, più specificamente, vi sono nella legge elettorale alcuni congegni che ci aiutano a comprendere ancor più gli effetti dell'interdipendenza tra la competizione nei collegi e quella nelle circoscrizioni e, soprattutto, come sia possibile che nei collegi uninominali la bilancia delle strategie competitive dei singoli partiti non penda tutta dal lato della scelta aggregativa. Congegni che, in definitiva, rappresentano un'attenuazione dell'effetto maggioritario dovuto all'esistenza di collegi uninominali in numero pari al $75 \%$ dei seggi complessivi.

Il più importante tra essi è lo scorporo, o meglio quella particolare norma dello scorporo che prevede la detrazione di voti pro quota nel caso di più liste collegate al candidato vincente in sede di collegio uninominale. Lo scorporo, in generale, è un meccanismo che consente ai partiti minori - quelli che non sono in grado di far eleggere i propri candidati di collegio - di usufruire di una maggiore quota di seggi proporzionali. Con lo scorporo pro quota, però, i partiti collegati ad un candidato di collegio vincente si vedono addebitato un costo in ragione dei voti proporzionali da essi ottenuti. In questo modo essi subiscono una riduzione della loro cifra elettorale circoscrizionale con la quale concorrono alla ripartizione proporzionale dei seggi. In altre parole, pagano il loro appoggio al candidato vincente di un altro partito con meno seggi per i propri candidati di lista. In realtà, è plausibile che questo meccanismo sia stato introdot-

${ }^{21}$ Ciò può esser vero sia per gli elettori che per gli stessi partiti in vista di possibili alleanze. Vi è da dire, comunque, che l'attuale contingenza politica, contrassegnata dalla destrutturazione del sistema partitico e dal venir meno dei vecchi legami di fedeltà, potrebbe costituire un contrappeso di tale effetto. 
to per incentivare i partiti che intendono collegarsi ad un unico candidato a considerarlo a tutti gli effetti come un candidato comune. Sta di fatto, però, che nell'attuale situazione politica italiana è difficile immaginare la presentazione di questo tipo di candidature, soprattutto in conseguenza delle divisioni alimentate (tra l'altro) da quasi cinquant'anni di proporzionale e ancor più a causa del voto di lista per il quale quegli stessi partiti concorrono separatamente. Ciò rende quel meccanismo, contrariamente alle probabili intenzioni dei legislatori, un disincentivo alle aggregazioni. I partiti che valutano la possibilità di non esprimere propri candidati in un determinato collegio, infatti, saranno disposti a collegarsi ad uno altrui solo se ricompensati in qualche modo: se non lo fossero ricaverebbero dall'accordo la perdita di una quota di voti, che avrebbero potuto invece utilizzare ai fini della ripartizione proporzionale dei seggi, senza ottenere alcun vantaggio. Lo scorporo pro quota, dunque, di per sé scoraggia un appoggio gratuito (senza contropartite) a candidature altrui. Ne consegue che le aggregazioni risultano dipendenti più dalla contrattazione di pagamenti collaterali che non dalla comune intenzione di sostenere un certo candidato.

Tali pagamenti, per di più, sono difficili da negoziare. In teoria, la loro forma più semplice è quella di un accordo tra i partiti su una rete di collegamenti incrociati verso $i$ rispettivi candidati. Questo accordo dovrà tener conto della forza relativa dei partiti che lo attuano. Ma può accadere che alla proporzionale compartecipazione delle detrazioni di voto non corrisponda un'eguale affermazione dei rappresentanti dei singoli partiti. Si valuti il seguente esempio. Un piccolo partito nazionale che stipula un'alleanza elettorale con un partito più grande, in base alla quale si attua un duplice collegamento verso $i$ rispettivi candidati di collegio, deve tener conto delle prevedibili defezioni connesse alla convergenza dell'elettorato del partito maggiore sui propri candidati - tanto più se l'alleanza è su scala nazionale. Defezioni che impedirebbero la loro elezione, e che comunque risulterebbero molto più decisive che non a parti invertite. $\mathrm{E}$, se così fosse, quel piccolo partito - che a livello di lista potrebbe comunque aver superato la soglia del $4 \%$ - avrebbe meno seggi proporzionali senza aver ottenuto in cambio seggi uninominali, nonostante l'appoggio riservato ai propri candidati di collegio da parte del partito più grande. A queste condizioni - e può essere una circostanza verosimile nell'attuale con- 
testo politico italiano ${ }^{22}$ - non vi sarebbe alcun incentivo all'aggregazione, quantomeno a livello dell'intero territorio nazionale. Si tratta di un punto che merita di essere sottolineato: tale meccanismo incrementa il potere negoziale dei partiti minori, i quali - già sicuri di una affermazione (per quanto limitata) in sede di ripartizione proporzionale - possono avere interesse a collegarsi ai candidati espressi dai partiti maggiori solo in cambio di consistenti pagamenti collaterali. In definitiva, lo scorporo pro quota si configura come un disincentivo alle aggregazio$\mathrm{ni}$, perlomeno ad aggregazioni a carattere nazionale e politicamente coerenti.

Un ulteriore meccanismo che indebolisce la formazione di compatte alleanze elettorali afferisce alla proclamazione degli eletti grazie al voto di lista. I candidati perdenti nel collegio uninominale, in base alle proprie cifre individuali, entrano nella graduatoria di tutte le liste ad essi collegate ai fini del conseguimento dei seggi attribuiti proporzionalmente, nell'eventualità che alle liste medesime sia assegnato mediante tale riparto un numero di mandati superiore a quello delle candidature di lista. Potrebbe verificarsi, perciò, che l'esponente di un partito $\mathrm{X}$ venga eletto grazie al voto di lista del partito $\mathrm{Y}$ a lui collegato ${ }^{23}$. Si tratta di una evenienza piuttosto improbabile, ma comunque tanto più possibile quanto minore è il numero delle candidature di lista presentate; e, come vedremo, non mancano gli incentivi per i partiti a comportarsi in tal modo.

Le aggregazioni dei partiti a livello di collegi uninominali, dunque, non presentano solo vantaggi. Resta comunque il fatto che in molte circostanze una qualche forma di alleanza elettora-

22 La verosimiglianza con l'attuale situazione italiana è comprensibile immaginando che questo piccolo partito sia collocato all'estremità di un polo. Si faccia l'esempio che Rifondazione comunista valuti la possibiltà di un sostegno a candidature comuni con il PDS. In quei collegi uninominali in cui si presentano candidati di RC è probabile che la parte moderata dell'elettorato del PDS defezioni, ossia non voti per essi (bensì per candidati più «centristi» oppure si astenga) e ne impedisca così l'elezione. Al contrario, gli elettori di RC che non gradiscono i candidati del PDS non hanno soluzioni alternative: possono tutt'al più astenersi, nel qual caso però incrementerebbero le possibilità di vittoria di candidati più distanti (e, quindi, ancora più sgraditi). Si consideri, comunque, che la «decisività» degli elettorati in relazione ad una alleanza elettorale dipende oltre che dalla loro compattezza anche dalla loro dimensione (che, ceteris paribus, gioca a scapito del partito più piccolo).

${ }^{23}$ Il rischio che ciò accada è comunque maggiore per le liste più grandi. Infatti la proclamazione degli eletti in base alla graduatoria delle cifre individuali dei candidati di collegio non eletti e collegati a più liste procede a cominciare dalla lista con la cifra elettorale più elevata. 
le può essere decisiva. In tali casi - e a certe condizioni - è anche possibile che essa sia realizzata in modo più conveniente per i partiti, aggirando la disposizione di legge sui collegamenti e quindi il costo dello scorporo.

Una prima possibilità potrebbe essere quella di spartirsi $i$ collegi accordandosi affinché non vengano presentate candidature contrapposte: ognuno dei partiti alleati si «accontenta» di far concorrere (e di collegarsi a) propri esponenti in numero proporzionale alla propria forza relativa; negli altri collegi connessi all'accordo sosterrà (non collegandosi) le altrui candidature. Il vantaggio di questa operazione è quello di unire ai benefici del reciproco appoggio l'evasione dei costi associati al collegamento - lo scorporo pro quota (lo scorporo sarebbe a carico del solo partito cui il candidato vincente appartiene), e l'elezione di candidati sconfitti nel collegio uninominale grazie al voto di lista, ma di una lista che, per quanto collegata, non è la propria.

Un'altra soluzione - che si configura come un vero e proprio espediente - verte sulla possibilità di annullare del tutto i costi dovuti al meccanismo dello scorporo (anche per la lista di effettiva appartenenza del candidato di collegio vincente) ed ha implicazioni rilevanti anche nei confronti delle strategie (non aggregative) dei singoli (grandi) partiti. Ferma restando la volontà di sostenersi a vicenda e/o di presentare candidature comuni, le liste potrebbero esse stesse non collegarsi ai propri candidati creando una «lista fittizia» pensata proprio all'unico scopo del collegamento: questa sarebbe sì presente in sede di ripartizione proporzionale dei seggi ma solamente per accollarsi l'onere dello scorporo. Così come ora formulata, tale ipotesi è soggetta a numerose e convincenti obiezioni: 1) questa lista sarebbe in concorrenza proprio con quelle che la hanno artificiosamente realizzata; 2 ) il candidato collegato ad essa, se sconfitto nel collegio, perderebbe le chances di ottenere la rappresentanza grazie al possibile storno dei seggi non assegnati alle candidature di lista; 3) gli elettori che nel collegio uninominale votano comunque per il simbolo (e non per il nome), non troverebbero più il proprio; 4) l'ambigua natura di tale collegamento potrebbe disorientare il proprio elettorato a danno sia del candidato che delle liste che realmente - anche se non in base alla legge - lo appoggiano.

Indubbiamente tra queste obiezioni la più forte è la seconda: il collegio uninominale funzionerebbe realmente all'inglese, 
per cui, data la possibilità che non sia così, un certo numero di candidati non troppo sicuri della propria elezione in quella sede (i più) non sarebbe affatto d'accordo con tale ipotesi ${ }^{24}$. Ma questa strategia resta comunque valida per i candidati sicuri; ed anche se è probabile che in una contingenza fluida ed aleatoria di candidati sicuri non ve ne saranno molti, ve ne saranno comunque sempre di più una volta stabilizzati i rapporti di forza (e avviato, per quanto possibile, il processo di concentrazione degli schieramenti). Quanto alle altre obiezioni precedentemente sollevate ${ }^{25}$, esse prospettano disincentivi non determinanti, che potrebbero essere compensati dal beneficio di eludere lo scorporo. Infatti, in primo luogo, è evidente che la lista fittizia non riceverà alcuna forma di propaganda ai fini del voto nella circoscrizione. In secondo luogo, in relazione al contrassegno, se anche non fosse più possibile da parte del candidato venir affiancato dal simbolo della lista di effettiva appartenenza (poiché il collegamento diverrebbe automatico), nulla gli vieterebbe di adottarne uno che lo evochi ${ }^{26}$ o che, comunque, ne attesti sotto altre spoglie - l'identità di partito ${ }^{27}$. L'ipotesi della lista

24 Tanto più che per quei candidati verrebbero meno le chances connesse alla eventuale candidatura di lista. Infatti, come sarà chiaro più avanti, sarebbe un nonsense presentarla (potrebbe sviare i potenziali elettori del partito artefice della lista fittizia) e, ad ogni modo, tale lista è «a perdere», ossia non otterrebbe voti (se non per sbaglio).

${ }_{25}$ Alle quali se ne può aggiungere un'ulteriore. Se è vero, infatti, che l'eventuale uniformità di comportamento in direzione di tale ipotesi strategica - ossia della artificiosa creazione di liste fittizie - annullerebbe, distribuendoli a tutti proporzionalmente, i vantaggi, è vero anche che l'eventuale defezione da essa, in base alla logica inversa al free-riding, sarebbe pagata a caro prezzo - il prezzo corrispondente al totale degli oneri connessi allo scorporo cui si sono sottratte tutte le liste non collegate ai propri candidati vincenti nei collegi uninominali.

${ }^{26}$ La legge è molto puntuale affinché sia evitata la confondibilità dei contrassegni; $\mathrm{ma}$ in tale circostanza non verrebbe chiaramente avanzato alcun reclamo.

${ }^{27}$ Quanto al possibile disorientamento dell'elettorato, se possa costituire un'eventualità o meno dipende dall'esatta interpretazione della legge in merito alla corrispondenza del simbolo del candidato di collegio con quello della lista (o di almeno una delle liste) collegata/e. Sul punto la normativa non è del tutto chiara e bisogna, quindi, far professione di cautela. Se l'interpretazione esatta è che il contrassegno del candidato di collegio deve comunque corrispondere a quello della lista collegata (qui della lista fittizia) è evidente che in questo caso possano sorgere confusioni all'atto del voto per il livello proporzionale: il partito di effettiva appartenenza di quel candidato rischierebbe di perdere voti di propri elettori che si sono lasciati sviare dall'uniformità del simbolo. $\mathrm{Ma}$ se l'interpretazione autentica è quella per cui tale corrispondenza non è obbligatoria, allora l'ipotesi della lista fittizia si rafforza nella sua praticabilità: il candidato di collegio avrà ovviamente un contrassegno diverso da quello della lista fittizia a lui collegata, la quale sarà così, per gli elettori che hanno espresso il voto per quel candidato, una lista sconosciuta come tante altre (l'ufficialità del collegamento, infatti, non comparirebbe altro che agli atti del Ministero dell'Interno). Da cui, la lista fittizia non potrebbe in 
fittizia, in sostanza, potrebbe costituire una ulteriore possibilità strategica, sebbene limitata ai collegi davvero sicuri, a disposizione dei partiti ${ }^{28}$.

In conclusione, quello che emerge da questa analisi è che la competizione tra i partiti tende ad essere rispondente per alcuni aspetti ad una logica maggioritaria (di aggregazione), ma per altri ancora ad una logica proporzionalistica (di differenziazione). Le alleanze elettorali, infatti, seppur incentivate non lo sono in maniera decisiva. Per i motivi addotti non è scontata la realizzazione di accordi nazionali a carattere strategico (politico-programmatici e/o politico-ideologici). Viceversa, è possibile aspettarsi alleanze elettorali tattiche, attuate in determinate circoscrizioni e non in altre, magari facendo ricorso anche a quegli escamotages che abbiamo ora illustrato. Tanto più che nella situazione italiana attuale tre fenomeni aggraverebbero queste tendenze: la concentrazione geografica del voto ${ }^{29}$, l'asimmetria delle eventuali aggregazioni e la distanza politico-ideologica che separa i partiti all'interno dei due possibili schieramenti alternativi.

\section{Meccanismi elettorali e strategie dei candidati}

Un sistema elettorale non è solo una struttura di incentivi per $i$ partiti, ma anche per $i$ candidati. Così come i partiti, anche i candidati sono costretti a calcolare gli effetti dei meccanismi del sistema sulla loro probabilità di elezione e quindi a scegliere la strategia ottimale per essere eletti tra tutte quelle disponibili. Nei sistemi proporzionali strategie dei partiti e strategie dei candidati tendono a coincidere perché la funzione-obiettivo dei partiti - la massimizzazione del voto - non è in conflitto con la funzione-obiettivo del candidato - la massimizzazione della sua probabilità di elezione. Quindi possiamo dire che in tali sistemi l'interesse del partito e quello del candidato sono comuni. Non è sempre così con altri tipi di sistema elettorale.

alcun modo fare concorrenza al partito che la ha creata. Ad ogni modo, devono essere tenuti presenti i costi «politici» di tale operazione, i quali ne possono limitare l'utilizzo.

${ }^{28} \mathrm{E}$, come già accennato, indipendentemente dalla circostanza che ad utilizzarla siano singoli (grandi) partiti o aggregazioni di più liste.

${ }^{29} \mathrm{Ci}$ riferiamo non soltanto alla posizione della Lega nel Nord Italia, ma anche a quella - così come ora appare - del PDS al Centro, della DC e dell'MSI al Sud. 
In quello che stiamo analizzando, poi, le cose sono ancora più complesse data la presenza di due livelli di competizione che rispondono a regole diverse ma che - non ci stancheremo di sottolinearlo - sono interdipendenti tra loro.

Il nostro sistema offre ai potenziali candidati alla Camera (non così al Senato) tre diverse strategie elettorali:

1. presentarsi soltanto nel collegio uninominale;

2. presentarsi soltanto in una lista circoscrizionale;

3. presentarsi in entrambe le sedi.

Una ulteriore possibilità è quella di presentarsi nella stessa lista (lista con lo stesso contrassegno) in più circoscrizioni (fino a un massimo di tre). Questa possibilità però non configura una vera e propria strategia elettorale. Almeno non nel senso che diamo qui a questo termine.

Tra le tre alternative indicate sopra, quale è la strategia ottimale per massimizzare la probabilità di elezione? Dati i meccanismi del sistema, tale strategia è sicuramente la terza, soprattutto se il candidato riuscirà ad occupare la prima posizione nella lista circoscrizionale. Infatti, in caso di sconfitta nel collegio, l'elezione può essere assicurata dalla quota proporzionale. Un candidato che riuscisse ad attuare una simile strategia si pone in una situazione privilegiata rispetto agli altri candidati del suo stesso partito. In altre parole, non è in competizione con nessuno di loro in quanto la competizione con essi l'ha già vinta prima delle elezioni. Questa opportunità però non è disponibile per tutti i candidati di collegio. Anche ammesso che il partito scelga di presentare nelle liste $i$ candidati dei collegi, invece di differenziare nettamente le due sedi, non potrebbe accontentarli tutti visto che le posizioni da assegnare sono al massimo 4 e nella maggior parte delle circoscrizioni anche meno (un terzo dei seggi proporzionali). In ogni caso, si creerebbe così un elemento di competizione pre-elettorale all'interno dell'insieme dei candidati di collegio dello stesso partito. Le altre due strategie, che ora andremo ad analizzare, configurano invece una situazione di conflitto potenziale e quindi di competizione tra candidati dello stesso partito anche a livello elettorale.

Vediamo la prima strategia. In questo caso, va da sé che l'elezione dipende dal numero dei voti che ciascun candidato riuscirà a raccogliere da solo $o$ in collegamento con altre liste oltre alla sua. La politica delle alleanze assume quindi un ruolo 
cruciale soprattutto per candidati di partiti piccoli e medi. $\mathrm{Ma}$ le possibilità non finiscono qui. Il sistema, infatti, prevede il recupero dei candidati di collegio perdenti a condizione che il numero dei seggi assegnati in sede proporzionale sia superiore al numero dei candidati indicati nella lista circoscrizionale. In questo caso vincono il seggio quei candidati sconfitti nei collegi uninominali che hanno la più alta cifra individuale. Così stando le cose, al candidato uninominale conviene o apparire in prima posizione anche nella lista cui è collegato (è la strategia già analizzata sopra) oppure che la lista sia la più «corta» possibile e, magari, con un nome solo. Ma una tale richiesta pone in conflitto questa categoria di candidati con l'altra categoria, quella dei candidati di lista, il cui interesse ovviamente è quello di avere la posizione più alta possibile nella lista senza doverla negoziare con i candidati dei collegi.

Passiamo ora ad analizzare la seconda strategia. Questa strategia è l'unica rilevante per i candidati di partiti piccoli o medi, i quali, senza alleanze sufficientemente forti a livello di collegio, hanno la sola chance di successo nella quota proporzionale. Per i candidati di questi partiti non si pongono problemi particolari se non quello di occupare la posizione migliore nella lista. Per essi non esiste conflitto con i candidati di collegio visto che questi non hanno comunque nessuna possibilità di vincere. Semmai, il rischio da evitare è che il proprio partito si allei con altri a livello di collegi per far vincere candidati il cui eventuale successo verrebbe da essi stessi pagato attraverso il meccanismo dello scorporo pro quota; meccanismo che conferma così la sua natura di disincentivo alla aggregazione.

Le cose stanno diversamente per i partiti maggiori. In questo caso $\mathrm{i}$ candidati di lista sono direttamente in competizione con i candidati di collegio. È il meccanismo dello scorporo che determina questa situazione. Infatti, ogni vittoria di un candidato di collegio diminuisce il numero dei voti che servono alla assegnazione dei seggi proporzionali e quindi riduce le possibilità di elezione dei candidati di lista. Ne segue che ai candidati di lista non conviene affatto la vittoria di colleghi dello stesso partito che si presentano nei collegi uninominali. Ad essi conviene invece che in tali collegi vengano presentate candidature deboli, con scarse probabilità di successo, per garantirsi il maggior numero di voti proporzionali. Va da sé che l'interesse del partito in questo caso non coincide con l'interesse dei suoi candidati di lista. Questi invece hanno un vero e proprio incentivo a dire ai 
propri elettori di non votare i candidati di collegio del proprio partito ${ }^{30}$.

Questa situazione è particolarmente delicata se assumiamo che $\mathrm{i}$ candidati di collegio siano - per usare un termine in voga - «espressione della società civile» e i candidati di lista siano membri dell'apparato. Il candidato-apparato che mantiene la possibilità di essere eletto a livello circoscrizionale, ma non a livello di collegio, ha tutto l'interesse a impedire che il partito presenti nei collegi candidati-espressione della società civile che abbiano possibilità di vincere.

A quest'ultima osservazione, si potrebbe rispondere che la presentazione di candidature attraenti tende a portare voti alla lista e quindi ad aumentare, nonostante lo scorporo, il numero dei voti utili per l'assegnazione dei seggi proporzionali (effetto bandwagon o effetto di trascinamento). Questo è vero, però, soltanto se assumiamo che gli elettori votino nello stesso modo sia nel collegio che nella circoscrizione. Ma ciò non è affatto detto, visto che le schede a disposizione dell'elettore sono due. Anzi, uno degli sviluppi più interessanti da seguire nell'applicazione di questo sistema elettorale sarà proprio la diffusione del voto diviso (o differenziato). Quanti elettori voteranno per un candidato nel collegio e per una lista non collegata a quel candidato nella circoscrizione?

Un'ultima riflessione riguarda i rapporti all'interno del gruppo dei candidati di uno stesso partito che si presentano nei collegi della circoscrizione. Questi candidati non solo hanno interesse - come gruppo - che la loro rispettiva lista circoscrizionale sia la più corta possibile, ma hanno anche un interesse preciso che nessuno di loro vi compaia. $E$, in ogni caso, $i$ candidati più deboli - vale a dire quelli di collegi insicuri - hanno interesse che nella lista compaiano solo i candidati forti - quelli cioè sicuri vincitori nei rispettivi collegi. In sostanza, possiamo quindi pensare a due categorie di candidati di collegio, quella dei sicuri (forti) all'interno della quale non esiste concorrenza, e quella degli insicuri (deboli) per $\mathrm{i}$ quali invece si prospetta una situazione competitiva al loro interno. Infatti, a condizione che nessuno di essi compaia nella lista circoscrizionale e che tale li-

30 Tale effetto è comunque attenuato dalla circostanza che i candidati di lista sono pochi: tutt'al più in numero pari ad $1 / 3$ dei seggi proporzionali della circoscrizione e, quindi, a circa $1 / 9$ dei candidati di collegio della propria lista (se presente ovunque). 
sta sia corta, l'eventuale vittoria (mediante recupero in sede proporzionale) dipende dalle loro cifre individuali, ammesso che la loro lista abbia a livello nazionale e a livello circoscrizionale un numero di voti sufficiente a garantire uno o più seggi. In questo caso il voto nel collegio funzionerebbe in pratica come un voto di preferenza.

La strategia della lista corta, associata alla norma sul recupero delle candidature nei collegi, favorisce dunque la competizione intrapartitica. Essa mette sullo stesso piano tutti i candidati nei collegi incrementando il loro interesse a competere e a investire nella propria elezione anche quando non hanno nessuna possibilità di vincere il seggio uninominale. Un secondo effetto di questa strategia è quello di una possibile trasformazione dei collegi uninominali in collegi plurinominali: può accadere, infatti, che un collegio esprima insieme al candidato eletto con il sistema maggioritario anche uno o più candidati di altri partiti che, pur avendo perso in quel collegio, vengono recuperati grazie alla quota proporzionale ${ }^{31}$. Un terzo effetto è quello di consentire l'aggiramento della previsione di legge relativamente alla presentazione nelle liste circoscrizionali di candidati e candidate in ordine alternato.

\section{Il comportamento degli elettori: voto diviso, voto sincero e voto strategico}

In un sistema elettorale come quello creato dalla nuova legge per la Camera, come si dovrebbe comportare il singolo elettore che volesse usare il suo voto per massimizzare la probabilità che il seggio nel collegio e i seggi nella circoscrizione vadano al candidato/i preferito/i? Esiste una strategia ottimale per l'elettore razionale ${ }^{32}$ ? Quali sono le variabili che la determinano? Sono queste le domande cui cercheremo di rispondere in questa sezione. Va da sé che ci poniamo queste domande per-

31 Questo effetto si può produrre, oltre che nel caso della lista corta, anche quando ad un partito tocchino più seggi proporzionali del numero massimo di candidati di lista.

32 Senza entrare in un'analisi specifica del concetto di razionalità, definiamo in questa sede razionale l'elettore il cui obbiettivo è quello di massimizzare l'impatto del suo voto sull'esito della singola competizione elettorale. A questo tipo di elettore faremo riferimento anche con il termine di elettore di opinione, anche se i due concetti non sono necessariamente equivalenti. 
ché postuliamo che gli elettori vogliano utilizzare il voto come strumento di scelta e non solo come espressione di appartenenza. In quale misura questo accada nel nostro sistema politico o in altri non è questione che affrontiamo in questa sede. Qui a noi interessa solo individuare gli incentivi offerti dal sistema elettorale ad un comportamento razionale.

In un sistema elettorale perfettamente proporzionale o quasi, l'elettore non ha problemi di scelta: il suo voto va al partito o al candidato preferito in assoluto. Infatti, assumiamo che l'elettore abbia una scala di preferenza (una graduatoria) dei candidati/partiti in lizza e che in questa graduatoria essi compaiano in ordine dal più preferito $\mathrm{al} \mathrm{meno} \mathrm{preferito:} \mathrm{il} \mathrm{suo} \mathrm{voto} \mathrm{va}$ necessariamente al partito/candidato che si colloca in cima alla lista delle sue preferenze. In questo caso l'elettore esprime un voto sincero ${ }^{33}$. Né gli converrebbe votare diversamente. Infatti, dato il meccanismo proporzionale, il suo voto in ogni caso contribuisce all'elezione di candidati presenti nelle liste del partito preferito, salvo il caso in cui quel partito non ottenga alcun quoziente elettorale ${ }^{34}$.

In un sistema elettorale maggioritario/uninominale la situazione è più complessa perché l'elettore può scegliere fra due alternative di comportamento: votare in modo sincero o votare in modo strategico. Il voto strategico è il voto a un partito o a un candidato che l'elettore preferisce meno di altri, ma che ritiene abbia maggiori probabilità di successo di quello maggiormente preferito. Un comportamento strategico è quindi frutto di un calcolo da parte dell'elettore. In un collegio uninominale dove vige la regola della maggioranza relativa, l'elettore che ritiene perdente il proprio candidato avrebbe interesse a votarne un altro con maggiori probabilità di vittoria invece di «sprecare» il suo voto attribuendolo a quel candidato comunque destinato a non conquistare il seggio. Naturalmente, non è detto che l'elettore si comporti in modo strategico. Un elettore di appartenenza continuerà a votare sinceramente. È più probabile che sia l'elettore di opinione a votare in modo strategico. Ma questa è una questione da valutare sul piano empirico. Il punto da sottolineare in questa sede è che il sistema elettorale che stiamo ana(1969).

33 Riguardo ai concetti di voto sincero e voto strategico si veda Farquharson

${ }_{34}$ Questo ragionamento è tanto più valido quanto più il sistema proporzionale è puro. 
lizzando offre degli incentivi al voto strategico e quindi «obbliga» l'elettore che vuole massimizzare l'utilità attesa del suo voto a calcolarne i probabili effetti. Questo, da un punto di vista sistemico è un vantaggio, perché partiti e candidati devono tener conto nelle loro strategie competitive anche delle seconde preferenze degli elettori. Ma il vantaggio viene meno se il sistema elettorale è talmente complesso da rendere difficile da parte dell'elettore l'espressione di un voto strategico.

Ciò premesso, in quali casi conviene ad un ipotetico elettore di opinione votare sinceramente e in quali casi votare strategicamente? Parliamo di elettore di opinione perché assumiamo che l'elettore di appartenenza comunque voti sinceramente anche a costo di «sprecare» il suo voto. Ciò non toglie però alcuna validità alla conclusione che, così facendo, esso si comporta in modo tale da favorire l'elezione di candidati meno preferiti o più sgraditi di altri. Per rispondere a queste domande, in riferimento al sistema elettorale per la Camera dei deputati, occorre distinguere i due livelli del voto - il collegio e la circoscrizione - che corrispondono alle due schede e quindi ai due voti che l'elettore ha a disposizione. Diciamo subito infatti che le ipotesi riferite ad un livello non necessariamente si applicano al secondo. Inoltre, è assai probabile, date le caratteristiche del sistema (e la situazione politica attuale), che si manifesti in maniera significativa il fenomeno del «voto diviso», vale a dire che anche l'elettore sincero voti per partiti diversi nel collegio e nella circoscrizione.

Cominciamo dal collegio uninominale e dall'analisi di quei casi in cui all'elettore conviene votare sinceramente.

Caso 1 . Il candidato maggiormente preferito è il candidato potenzialmente vincente o comunque è un candidato competitivo, vale a dire con ragionevoli speranze di vittoria. Non esiste strategia migliore che votare questo candidato.

Caso 2. Il candidato maggiormente preferito non è competitivo (le sue possibilità di elezione nel collegio sono scarse o nulle), ma soddisfa queste condizioni:

a) appartiene ad una lista che supera il $4 \%$ dei voti validi in sede nazionale;

b) la sua lista di appartenenza ha la possibilità di ottenere più di un quoziente elettorale circoscrizionale;

c) la lista circoscrizionale è «corta»;

d) il suo nome non compare sulla lista circoscrizionale.

Date queste condizioni, all'elettore conviene comunque votare questo candidato invece di un altro perché il suo voto va 
ad aumentarne la cifra individuale e quindi la possibilità di elezione attraverso il meccanismo del recupero dei candidati perdenti nei collegi, qualora i seggi assegnati alla lista siano più dei candidati iscritti.

Da questo secondo caso si passa facilmente a costruire delle ipotesi di voto strategico. La variabile chiave resta la competitività del candidato maggiormente preferito. Se questo candidato non è competitivo, la strategia ottimale dell'elettore è quella di votarne un altro che sia meno preferito del candidato ottimale, ma più preferito del candidato potenzialmente vincente. Così facendo egli contribuisce ad eleggere un candidato meno sgradito o comunque ad impedire l'elezione del «candidato peggiore». Se è indifferente tra $\mathrm{i}$ candidati con maggiori possibilità di successo, o vota sinceramente o si astiene. Questo è il quadro di scelta dell'elettore nei sistemi maggioritari/uninominali puri. $\mathrm{Ma}$ il nostro non appartiene a questa categoria, per cui all'elettore potrebbe convenire votare sinceramente anche in alcuni casi in cui il suo candidato ottimale non sia competitivo nel collegio.

Partendo dal caso 2 illustrato sopra e modificando una qualunque delle condizioni ivi enunciate si individuano $i$ casi in cui all'elettore conviene votare strategicamente. In particolare, è più probabile che ciò avvenga tutte quelle volte in cui il candidato ottimale è collegato ad una lista circoscrizionale «lunga» per cui le sue possibilità di recupero sono nulle. Oppure quando il candidato appartiene ad un partito che non ha nessuna speranza di superare la soglia del $4 \%$ a livello nazionale. A queste condizioni un voto dato al candidato preferito, ma comunque perdente, è, nell'ottica di questa analisi, un voto «sprecato» ${ }^{35}$.

Un altro caso di convenienza del voto strategico, che merita sottolineare, è dato dalla presenza simultanea di queste condizioni:

a) il candidato ottimale nel collegio non è competitivo;

b) il suo nome appare in cima alla lista circoscrizionale cui è collegato;

c) la lista cui è collegato ha la possibilità sia di superare la soglia del $4 \%$ che di ottenere almeno un quoziente elettorale circoscrizionale.

35 Anche il raggiungimento del quoziente circoscrizionale da parte del partito cui appartiene il candidato preferito potrebbe essere previsto. In tal caso, però, i calcoli che dovrebbe fare l'elettore sono molto più complessi e quindi irrealistici, mentre nelle due ipotesi che abbiamo prospettato è ragionevole assumere che una quota di elettori di opinione si comporti in modo tale da calcolare l'effetto del proprio voto sul risultato finale. 
In questo caso l'elezione del candidato ottimale sarebbe assicurata comunque dal voto di lista (corrispondente alla seconda scheda), per cui è più conveniente utilizzare la prima scheda per votare in maniera strategica. Per esempio votando quello dei due candidati più competitivi che piace maggiormente, in modo da ottenere almeno uno di questi risultati: 1) aver contribuito all'elezione di un candidato più preferito o meno sgradito nel caso in cui il candidato votato vinca, o 2) aver aumentato il costo della vittoria per il partito del candidato vincente qualora il candidato votato arrivi secondo. Quest'ultimo effetto è il risultato del meccanismo dello scorporo. In realtà, questo meccanismo offre un notevole incentivo al voto strategico. Infatti, tutte le volte in cui l'elettore si trova di fronte un candidato preferito che non sia competitivo, gli conviene doppiamente votarne un altro che abbia più probabilità di vincere o comunque di arrivare secondo, perché in questo modo aumenta le possibilità che la sua lista circoscrizionale preferita abbia più seggi.

Fin qui abbiamo trattato soprattutto del voto nel collegio.

Quale è la situazione a livello di circoscrizione? Trattandosi di un voto proporzionale, non sorprende che le scelte dell'elettore siano più limitate. Nella stragrande maggioranza dei casi all'elettore conviene votare sinceramente, cioè per la lista preferita. Sono due le eccezioni a questa regola di comportamento. La prima ha a che vedere con la soglia del $4 \%$. Va da sé che, se il partito preferito dal nostro elettore razionale è destinato a non superare questa soglia, un voto dato ad esso è un voto sprecato. Lo stesso dicasi in riferimento al mancato raggiungimento del quoziente elettorale circoscrizionale da parte del partito maggiormente preferito.

Quali conclusioni possiamo trarre da questa analisi delle scelte dell'elettore? Il nuovo sistema non facilita l'espressione di un voto razionale. Troppe sono le variabili in gioco che possono influenzare l'esito della competizione perché un elettore di opinione possa ragionevolmente calcolare il probabile impatto del suo voto. Ciò non deriva tanto dalla scelta del mix maggioritario-proporzionale, quanto dalle modalità di interconnessione tra i due tipi di sistema, ovvero tra i due livelli decisionali: il collegio e la circoscrizion $e^{36}$. By default, è probabile quindi che la maggior parte degli elettori di opinione voti comunque sinceramente, anche in quelle circostanze in cui un voto strategico

36 Un sistema maggioritario puro oppure un sistema a due turni presentano difficoltà minori all'elettore. 
servirebbe a far eleggere un candidato maggiormente preferito (o comunque meno sgradito) e quindi a produrre un risultato più rispondente alla propria struttura di preferenze. Votare sinceramente non sempre conviene con questo sistema elettorale, ma votare in modo strategico richiede in molti casi un livello di informazioni e una capacità di analisi che si possono attribuire a pochi elettori. Nella misura in cui questo fenomeno si verificherà, potremo allora dire che la complessità del sistema tende a ridurre la corrispondenza tra preferenze dell'elettorato e composizione della rappresentanza politica, anche al di là degli effetti propri del sistema maggioritario-uninominale.

Resta da dire però che, presumibilmente, non saranno poche le situazioni in cui molti elettori scopriranno l'utilità di votare candidati che con un sistema proporzionale, come il precedente, non avrebbero votato. Da questo punto di vista, il nuovo sistema elettorale indurrà gli elettori a informarsi di più e a prendere in considerazione nelle loro decisioni di voto anche altri candidati oltre a quelli maggiormente preferiti, vale a dire le loro seconde e terze preferenze, rendendo così il mercato elettorale più aperto e più dinamico. Se questo fenomeno si verificasse all'interno di un sistema elettorale dove fossero determinanti gli incentivi alla aggregazione tra i partiti, questo comportamento sarebbe sicuramente funzionale alla ridefinizione dello spazio politico e quindi alla ristrutturazione del sistema partitico. Nel nostro caso, e nella attuale fase di transizione della democrazia italiana, è poco probabile, però, che questo effetto si verifichi. Quantomeno in tempi brevi.

\section{Le differenze tra Camera e Senato}

Il principio ispiratore della riforma per il Senato è lo stesso di quello per la Camera: la creazione di un sistema elettorale misto con il $75 \%$ dei seggi assegnati in collegi uninominali in base alla regola della maggioranza relativa e il restante $25 \%$ assegnato a livello di circoscrizioni regionali in ragione proporzionale tra i gruppi di candidati concorrenti nei collegi uninominali.

A ciascuna regione è assegnata una quota di seggi in proporzione agli abitanti ${ }^{37}$. Il territorio di ogni regione, con ecce-

37 Nel rispetto, comunque, del vincolo dettato dall'art. 57 della Costituzione, il quale prevede che a ciascuna regione (tranne Molise e Valle d'Aosta) siano attribuiti non meno di 7 senatori. 
zione del Molise e della Valle d'Aosta, è diviso in collegi uninominali in numero pari a tre quarti dei seggi spettanti, con arrotondamento per difetto. I restanti seggi sono attribuiti mediante riparto proporzionale a livello dell'intera circoscrizione. La Valle d'Aosta è costituita in un unico collegio uninominale e il Molise in due (Tab. 3).

TAB. 3. Circoscrizioni regionali

\begin{tabular}{|c|l|r|r|c|}
\hline$n$. & Circoscrzioni regionali & Seggi tot. & Seggi unin. & Seggi prop. \\
\hline 1 & Piemonte & 23 & 17 & 6 \\
2 & Valle d'Aosta & 1 & 1 & 0 \\
3 & Lombardia & 47 & 35 & 12 \\
4 & Trentino-Alto Adige & 7 & 6 & 1 \\
5 & Veneto & 23 & 17 & 6 \\
6 & Friuli-Venezia Giulia & 7 & 5 & 2 \\
7 & Liguria & 9 & 6 & 3 \\
8 & Emilia-Romagna & 21 & 15 & 6 \\
9 & Toscana & 19 & 14 & 5 \\
10 & Umbria & 7 & 5 & 2 \\
11 & Marche & 8 & 6 & 2 \\
12 & Lazio & 28 & 21 & 7 \\
13 & Abruzzi & 7 & 5 & 2 \\
14 & Molise & 2 & 2 & 0 \\
15 & Campania & 30 & 22 & 8 \\
16 & Puglia & 22 & 16 & 6 \\
17 & Basilicata & 7 & 5 & 2 \\
18 & Calabria & 11 & 8 & 3 \\
19 & Sicilia & 27 & 20 & 7 \\
20 & Sardegna & 9 & 6 & 3 \\
\hline Tot. & Italia & 315 & 232 & 83 \\
\hline
\end{tabular}

Ciò premesso, occorre subito dire che le differenze tra i due sistemi, che pure derivano dallo stesso principio, sono significative $^{38}$. Le più importanti, a nostro avviso, sono queste:

1. la previsione di una unica categoria di candidati, quelli di collegio, e quindi l'assenza di liste a livello di circoscrizione regionale;

2. il voto unico espresso in una unica scheda, e quindi l'impossibilità del voto disgiunto tra livello maggioritario e livello proporzionale;

38 Queste differenze sono in gran parte dovute al fatto che il sistema elettorale per il Senato è stato condizionato più direttamente dal risultato del referendum elettorale del 18 aprile 1992. Mentre il sistema per la Camera, in mancanza di un testo «referendario», ha lasciato più ampi margini di manovra ai legislatori. 
3. il calcolo dello scorporo che viene fatto sulla base dei voti ricevuti dai candidati vincenti, e non dai secondi; quindi, scorporo totale e non parziale;

4. il meccanismo di determinazione della quota proporzionale che viene applicato su base regionale col metodo d'Hondt e non su base nazionale col metodo del quoziente naturale;

5. l'assenza di una soglia legale per accedere alla ripartizione dei seggi su base regionale e, al contrario, l'esistenza di una soglia di fatto il cui livello può variare da regione a regione e da un'elezione all'altra;

6. la possibilità di candidature indipendenti, cioè non collegate a nessun gruppo a livello regionale;

7. l'esistenza di un unico contrassegno a fianco del nome del candidato.

Queste differenze determinano una diversa struttura di incentivi al comportamento di elettori, candidati e partiti rispetto a quella che abbiamo descritto per la Camera. Questa diversità deriva sia dal livello della soglia di accesso alla ripartizione proporzionale dei seggi, sia dalle prime tre differenze elencate sopra. Per la Camera abbiamo visto come le maggiori opportunità strategiche offerte ai vari attori dipendano da quei meccanismi che legano i due livelli del voto (il collegio e per la circoscrizione). Gli effetti di tale interconnessione nel caso del Senato sono stati fortemente attenuati. Infatti, non esiste al Senato un vero e proprio livello circoscrizionale (regionale). I candidati nel collegio e nella circoscrizione sono gli stessi. Il voto è unico. L'elettore che vota un certo candidato in un dato collegio contribuisce sia alla sua elezione nel collegio che al suo eventuale recupero in sede proporzionale nel caso in cui questi non venga eletto nel collegio stesso, alla condizione che il partito cui è collegato superi la soglia di rappresentanza.

Il meccanismo appena descritto, fondato sull'assenza del doppio voto, semplifica le scelte dell'elettore razionale. Il voto diviso ovviamente non è più una opzione, mentre la scelta fra voto strategico e voto sincero diventa più facile. Infatti, le variabili di cui l'elettore deve tener conto per calcolare gli effetti del suo voto si riducono sostanzialmente a due: 1) l'aspettativa di successo del candidato maggiormente preferito (quella che abbiamo definito come competitività della candidatura); 2) l'aspettativa del superamento della soglia di rappresentanza per il partito cui il candidato preferito appartiene ${ }^{39}$.

${ }^{39}$ In entrambi i casi la disponibilità di sondaggi attendibili aiuterebbe l'elettore a 
Una volta calcolato il valore di queste due variabili, l'elettore ha tre scelte sufficientemente chiare: 1) votare per il suo candidato ottimale (prima preferenza) se questo è competitivo nel collegio; 2) votare per questo candidato anche quando non è competitivo, a condizione che il partito cui appartiene abbia buone possibilità di superare la soglia minima di rappresentanza; 3) votare per un altro candidato meno preferito del candidato ottimale, ma sicuramente più preferito di uno dei candidati potenzialmente vincenti, nel caso in cui il candidato ottimale non sia competitivo e appartenga ad un partito senza possibilità di superare la soglia. Rispetto ad un sistema maggioritario-uninominale puro, questo sistema offre una possibilità in più, l'opzione numero 2 . La ragione è che i candidati perdenti nei collegi sono quelli che vengono poi recuperati in sede di assegnazione dei seggi proporzionali. D'altronde non si può fare a meno di notare che questo meccanismo attenua l'effetto del «vincitore pigliatutto» (winner takes all) proprio dei sistemi maggioritari-uninominali e, come già notato, può trasformare in un certo senso collegi uninominali in collegi plurinominali.

Rispetto alla Camera, la semplificazione maggiore riguarda le strategie dei candidati. Infatti, per i candidati scompare del tutto la scelta tra presentarsi nel collegio e/o nella circoscrizione. La strategia ottimale è quella di candidarsi nel collegio più sicuro. Una possibilità alternativa consiste nel candidarsi anche in collegi non sicuri, a condizione che la quota di voti attesa sia tale da garantire il successo attraverso il repêchage proporzionale. Ciò vuol dire che il candidato deve essere in grado di valutare quanti voti può raccogliere in un certo collegio rispetto a quelli che possono raccogliere i suoi rivali di partito negli altri collegi. Da questo punto di vista il voto maggioritario diventa anche voto di preferenza. In ogni caso, è plausibile sostenere che il sistema per il Senato spinge i candidati a massimizzare comunque il voto per sé e contemporaneamente per il partito cui sono collegati. Non è così invece alla Camera, come abbiamo già visto.

ridurre i margini di incertezza nelle sue scelte e quindi faciliterebbe un comportamento razionale. Questo significa che il divieto puro e semplice alla effettuazione di sondaggi e/o alla pubblicizzazione dei loro risultati nel periodo immediatamente precedente la data delle elezioni non è sempre funzionale al corretto svolgimento della campagna elettorale. Soprattutto nel caso di sistemi maggioritari. Il vero problema è quello della regolamentazione del loro uso. 
Quanto ai partiti, la valutazione degli effetti del sistema verte soprattutto sull'efficacia della soglia di accesso al livello proporzionale come incentivo istituzionale alla aggregazione. Noi sappiamo che, data l'esistenza di questo livello di attribuzione di una quota dei seggi, una maggior o minore selettività della soglia determina una maggiore o minore tendenza alla aggregazione tra i partiti, ceteris paribus.

Quale è dunque il livello della soglia al Senato? Alla Camera tale livello è fissato per legge al $4 \%$ su scala nazionale; al Senato non esiste una soglia legale di questo tipo: esiste invece una soglia di fatto che dipende da alcuni fattori. I primi due sono: 1) la ripartizione dei seggi in sede regionale e non nazionale; 2) l'uso del metodo d'Hondt. Dati questi meccanismi, la variabile più importante che influenza il livello della soglia è la dimensione delle circoscrizioni regionali. Come si può vedere dalla Tab. 3, queste circoscrizioni sono piccole: si va da 1 a 12 seggi proporzionali, con una larghissima maggioranza di regioni compresa tra 1 e 6 . Questa variabile spinge la soglia verso l'alto. Infatti, essa è tanto più alta quanto minore è la dimensione delle circoscrizioni. Nel nostro caso però non possiamo fermarci qui e concludere tout court che la soglia per il Senato è sistematicamente e considerevolmente più alta di quella per la $\mathrm{Ca}$ mera. Infatti anche per il Senato è previsto il meccanismo dello scorporo e il suo funzionamento può modificarne il livello. Vediamo come.

Intanto occorre ricordare che al Senato, per i voti da sottrarre alla cifra elettorale di ciascun gruppo, si tiene conto del totale dei voti ottenuti dai candidati di collegio vincenti e non dei voti ottenuti dai candidati arrivati secondi ${ }^{40}$. Questo comporta un costo maggiore per $i$ partiti più forti che si traduce in un vantaggio a favore dei partiti medio-piccoli, per i quali si abbassa la soglia di rappresentanza, vale a dire il numero dei voti necessari ad ottenere almeno un seggio. Tale vantaggio, poi, risulta tanto maggiore quanto più alta è la concentrazione del voto sui candidati vincenti nei collegi uninominali. In altre parole, per i partiti medio-piccoli è più facile ottenere un seggio, ceteris paribus, laddove i partiti più grandi sono più forti. Para-

40 Questo elimina l'incentivo alla presentazione di candidature-civetta, presentate allo scopo di abbassare il numero dei voti del secondo arrivato e di ridurre così il costoscorporo per $i$ vincenti. 
dossalmente, quindi, maggiore è la concentrazione territoriale del voto, minori sono gli incentivi alla aggregazione.

A questo punto possiamo cercare di rispondere alla doman$\mathrm{da}$ da cui siamo partiti: è più alta alla Camera o al Senato la soglia di accesso alla ripartizione proporzionale dei seggi? In linea generale, si può affermare che sia più elevata la soglia al Senato. Questo dipende dalla forte influenza che su di essa ha la variabile «dimensione delle circoscrizioni». Però, non si può fare a meno di sottolineare che il livello della soglia non può essere determinato a priori per il motivo che esso dipende anche dall'effetto dello scorporo e da altre variabili quali il numero dei partiti e la distribuzione dei voti tra le liste perdenti. Ciò premesso, è possibile che alcuni partiti che, con una soglia nazionale del $4 \%$, non avrebbero ottenuto seggi alla Camera, possano ottenere uno o più seggi al Senato. Questo può accadere sia perché certi partiti piccoli potrebbero superare al Senato anche una soglia del $6-7 \%$ avendo un voto concentrato in certe regioni, sia perché la soglia al Senato potrebbe, date certe condizioni, essere anche inferiore al $4 \%$ della Camera (per esempio, in Lombardia).

Che dire dunque, in sintesi, delle differenze tra Camera e Senato? Non c'è dubbio che il sistema per il Senato sia meno complesso e più parsimonioso di quello per la Camera. Resta il fatto che è anch'esso un sistema misto e come tale soffre dei problemi connessi alla coesistenza di due logiche distinte. Anche qui la presenza di un livello di rappresentanza proporzionale può costituire un disincentivo ad aggregazioni partitiche politicamente omogenee ed estese all'intero territorio nazionale. Come abbiamo evidenziato nel caso della Camera, è possibile che la logica proporzionale «retroagisca» a livello maggioritario attraverso un effetto di spillover con il risultato di influenzare la dinamica della competizione elettorale anche in questa sede, al di là della consistenza stessa della quota di seggi proporzionali.

A differenza della Camera, però, è abbastanza probabile che il livello più elevato della soglia di rappresentanza, oltre all'assenza dello scorporo pro quota, offra comunque incentivi più forti ai piccoli partiti ad aggregarsi tra loro o ai maggiori. Il risultato potrebbe essere un sistema partitico meno frammentato rispetto a quello della Camera. Tuttavia non si può fare a meno di rilevare che, così come esiste un effetto di spillover del sistema proporzionale su quello maggioritario, potrebbe prodursi a livello del Senato un «effetto Camera», indipendentemente dalle caratteristiche specifiche del sistema elettorale del Senato. 


\section{Conclusioni}

Non è semplice trarre delle conclusioni generali sugli effetti di questo sistema elettorale. Non solo il sistema è molto complesso e farraginoso (soprattutto quello per la Camera), ma verrà applicato in una situazione politica estremamente fluida, caratterizzata da forte volatilità elettorale e da un notevole grado di destrutturazione dei partiti tradizionali. A ciò occorre, inoltre, aggiungere le difficoltà connesse all'apprendimento delle nuove regole da parte di partiti, candidati ed elettori. Sono questi fattori che, unitamente alla configurazione specifica del sistema elettorale, influenzeranno il formato e la meccanica del nuovo sistema partitico.

Di tali fattori non ci siamo occupati specificatamente in questo scritto, il cui scopo principale invece è quello di analizzare i meccanismi della nuova legge elettorale e i loro specifici effetti. Nel momento però di formulare delle conclusioni non si può non sottolineare che l'impatto complessivo di questo sistema elettorale sulla competizione politica e sulla rappresentanza parlamentare sarà il risultato della interazione tra i meccanismi che abbiamo analizzato e le variabili che caratterizzano il contesto in cui le nuove regole verranno applicate. Ciò premesso, resta il fatto che ogni sistema elettorale ha una sua autonoma capacità di influenzare i comportamenti degli attori nei processi elettorali, in quanto fissa un quadro di vincoli e di opportunità alla loro azione.

A noi sembra che il nuovo sistema elettorale ponga un freno alla crescita della frammentazione ${ }^{41}$, ma che non incentivi in maniera decisiva il crearsi di aggregazioni partitiche politicamente coerenti e a carattere nazionale e la formazione di uno schieramento bipolare. La proliferazione di piccoli partiti con un voto geograficamente disperso è certamente ostacolata dal livello della soglia di esclusione sia alla Camera che al Senato. Con questo sbarramento, dunque, si è sicuramente reso più difficile l'accesso al mercato elettorale per nuove formazioni politiche. Ma la soglia per la Camera è troppo bassa per assicurare una drastica riduzione della frammentazione esistente, almeno nel breve periodo. Se riduzione della frammentazione ci sarà, è più probabile che essa riguardi non tanto il numero dei partiti

41 Utilizziamo il termine di frammentazione nell'accezione attribuitagli da Rae $(1971,54-58)$ che ne individua due componenti: il numero dei partiti e la loro forza relativa. 
quanto, piuttosto, la dispersione del voto tra di essi, e dipenderà comunque dalla concentrazione geografica del voto intorno ai partiti maggiori e dalla loro sovrarappresentazione stante l'effetto distorsivo della componente maggioritaria del sistema. Nell'attuale sistema politico sono potenzialmente numerosi i partiti che possono ambire a superare la soglia da soli o formando mini-alleanze. I seggi a disposizione di questa strategia di sopravvivenza sono certo pochi, ma il fatto che ci siano può influenzare le scelte di questi attori. Tanto più che lo scorporo avvantaggia ulteriormente i partiti piccoli e medi che accedono alla rappresentanza. E al Senato che questa strategia potrebbe incontrare maggiori difficoltà, dato il livello più alto della soglia. Si tratterà di vedere se sarà prevalente la logica competitiva della Camera su quella del Senato o viceversa.

In teoria, un certo grado di frammentazione del sistema partitico non è incompatibile con il formarsi di uno schieramento bipolare che funzioni secondo una logica di competizione sistemica (D'Alimonte, 1989). Ma perché questo si verifichi occorre che il sistema elettorale contenga forti incentivi alla aggregazione e che siano soddisfatte determinate condizioni strutturali (Sartori, 1968). Nel caso italiano attuale, la concentrazione geografica del voto e la destrutturazione dei partiti tradizionali già di per sé rappresenterebbero seri ostacoli al manifestarsi di una tendenza bipolare e di una competizione di tipo sistemico anche se i meccanismi elettorali fossero fortemente aggregativi. La probabilità di un simile effetto si riduce poi ulteriormente tenendo conto che il nuovo sistema elettorale contiene meccanismi quali il doppio voto, il collegamento e lo scorporo (pro quota) che rappresentano semmai dei disincentivi ad accordi elettorali strategici e che favoriscono invece la realizzazione di accordi a carattere locale basati su compensazioni elettorali.

Né la formazione di alleanze coese e coerenti potrà contare sul comportamento degli elettori. Infatti, la presenza di due logiche distinte di voto, e la loro interdipendenza attraverso i meccanismi che abbiamo analizzato in questa sede, non facilita l'espressione di un voto che rifletta anche le loro seconde preferenze. Eppure, in una fase di ridefinizione dello spazio della politica italiana e di ristrutturazione del sistema partitico, sarebbe utile, da un punto di vista sistemico, che gli elettori potessero esprimere la loro opinione non solo sui candidati preferiti in assoluto, ma anche sugli altri in competizione in modo da influenzare e sanzionare essi stessi la formazione di alleanze di 
governo. Con questo sistema, e nelle attuali condizioni, questo ci sembra difficile.

\section{Riferimenti bibliografici}

Blais, A. (1988), The Classification of Electoral Systems, in «European Journal of Political Research», XVI, pp. 99-109.

D'Alimonte, R. (1989), Democrazia e competizione, in «Rivista Italiana di Scienza Politica», XIX, pp. 301-319.

Farquharson, R. (1969), Theory of Voting, New Haven, Yale University Press.

Fisichella, D. (1970), Sviluppo democratico e sistemi elettorali, Firenze, Sansoni.

Nohlen, D. (1989), Changes and Choices in Electoral Systems, in A. Lijphart, B. Grofman (a cura di), Choosing an Electoral System: Issues and Alternatives, New York, Praeger, pp. 217-224.

Rae, D.W. (1971), The Political Consequences of Electoral Laws, New Haven, Yale University Press.

Reeve, A. e A. Ware (1992), Electoral Systems. A Comparative and Theoretical Introduction, London, Routledge.

Sartori, G. (1968), Political Development and Political Engineering, in «Public Policy», vol. XVII; trad. it. Ingegneria politica e sistemi elettorali, in G. Sartori, Teoria dei partiti e caso italiano, Milano, Sugarco, 1982, pp. 97-128.

Taagepera, R. e M.S. Shugart (1989), Seats E Votes. The Effects and Determinants of Electoral System, New Haven and London, Yale University Press. 